\title{
Prevalencia de neuromitos en estudiantes de Pedagogía en Educación Física de Chile \\ Prevalence of neuromyths in students of Physical Education Pedagogy of Chile
}

Fernando Maureira Cid*, Elizabeth Flores Ferro**, Franklin Castillo-Retamal***, Manuel Enrique Cortés Cortés**, Sebastián Peña-Troncoso****, Valentina Bahamondes Acevedo*, Marcelo Hadweh Briceño*****,

Sonia Cárdenas Begazo**, Natalia Escobar Ruiz**, Benjamín Cortes Escaffi******

*Universidad Metropolitana de Ciencias de la Educación (Chile), **Universidad Bernardo O’Higgins (Chile), ***Universidad Católica del Maule (Chile), ****Universidad Austral de Chile (Chile), *****Universidad SEK (Chile), ******Universidad Católica Silva Henríquez (Chile)

Resumen. Introducción: la falta de un lenguaje común entre la neurociencia y la educación ha generado interpretaciones erróneas sobre el funcionamiento del sistema nervioso, dando origen a los neuromitos. El objetivo del presente estudio es describir la prevalencia de neuromitos y el conocimiento general de neurociencia de estudiantes de Pedagogía en Educación Física de diversas universidades de Chile. Metodología: la muestra estuvo constituida por 440 estudiantes de Educación Física de cinco universidades de Chile, a quienes se les aplicó la versión en español del Cuestionario de prevalencia de neuromitos desarrollado por Dekker et al. Resultados: los neuromitos más difundidos en esta muestra son los estilos de aprendizajeVAK, los efectos positivos de los entornos enriquecido en la edad preescolar y los ejercicios de coordinación para lograr la integración hemisférica, todos con una prevalencia de más del 90\%. En términos generales no existe diferencia en la prevalencia de neuromitos según sexo, curso de la carrera y la lectura habitual de textos científicos. Conclusión: los estudiantes de Educación Física de Chile presentan creencias en diversos neuromitos de forma similar a los reportados en estudiantes y profesores de otras pedagogías de otros países.

Palabras claves: neuromitos, educación física, estudiantes universitarios, neurociencia.

\begin{abstract}
Introduction: the lack of a common language between neuroscience and education has generated erroneous interpretations about the functioning of the nervous system, giving rise to neuromyths. The aim of this study is to describe the prevalence of neuromyths and the general knowledge of neuroscience of students of Physical Education Pedagogy from various Universities of Chile. Methodology: the sample was constituted by 440 students of Physical Education of five universities of Chile, to whom the Spanish version of the Questionnaire of prevalence of neuromyths developed by Dekker et al was applied. Results: The most widespread neuromyths in this sample areVAK learning styles and the positive effects of enriched environments in preschool age and coordination exercises to achieve hemispheric integration, both with a prevalence of more than $90 \%$. In general terms, there is no difference in the prevalence of neuromyths according to sex, course of study and regular reading of scientific texts. Conclusion: Physical education students from Chile present beliefs in various neuromyths similar to those reported in students and teachers of other pedagogies from other countries.
\end{abstract}

Keywords: neuromyths, physical education, university students, neuroscience.

\section{Introducción}

La neurociencia es un conjunto de disciplinas encargadas de estudiar la estructura del sistema nervioso y comprender cómo el funcionamiento de éste da origen a la conducta (Kandel et al., 2013), permitiendo con ello dilucidar procesos tan complejos como la atención, la memoria y el aprendizaje. Las implicancias de conocer cómo funciona el cerebro son enormes para una amplia variedad de disciplinas, desde la economía hasta la medicina, incluyendo la psicología, la sociología, el

Fecha recepción: 04-03-20. Fecha de aceptación: 21-04-21

Fernando Maureira

maureirafernando@yahoo.es marketing, el arte, las comunicaciones y la educación, por nombrar solo algunas (Arias, 2017, Markram, 2006, Maureira, 2018). Es en este último contexto donde Campos (2010) propone que la neurociencia es una herramienta para conocer de forma más amplia el cerebro, permitiendo comprender cómo se aprende, procesa, registra, conserva y evoca la información, para que a partir de este conocimiento se puedan mejorar las propuestas y experiencias de aprendizajes en la sala de clases. Aparicio (2009) explica que la neurociencia podría permitir eliminar algunos mitos en el proceso de aprendizaje, ayudando a superar prácticas inadecuadas y obsoletas. Sin embargo, en la actualidad existe una gran dicotomía entre los descubrimientos de la neurociencia y su interpretación y/o aplicación en las aulas (Varas \& Ferreira, 2017). 
La falta de un lenguaje común entre la neurociencia y la educación ha generado interpretaciones erróneas sobre el funcionamiento del sistema nervioso, dando origen a los neuromitos (OECD, 2002). Estas falsas creencias o conceptos erróneos sobre la mente han permeado a un gran porcentaje de profesores en todos los lugares del mundo, debido al gran atractivo de esta información, la cual, desafortunadamente, no es debidamente contrastada y es transferida desde los laboratorios de investigación hasta las escuelas sin las modificaciones necesarias (Geake, 2008). Esta situación ocasiona retrasos en el proceso de enseñanza-aprendizaje (PallarésDomínguez, 2016) y puede repercutir en pérdida de recursos (Carter, et al. 2020), ya que éstos son mal utilizados cuando la información en neurociencia que subyacería a ciertas creencias y prácticas en educación es imprecisa o errada (Varas \& Ferreira, 2017).

Entre los neuromitos más difundidos es posible mencionar: a) solo se usa el 10\% del cerebro, un mito difundido por la publicidad, el cine y los parapsicólogos, pero que no tiene una base real (OECD, 2002). En realidad, el cerebro permanece activo en todo momento, si bien cada región puede tener una actividad específica, durante el estado de vigilia e incluso el sueño, ningún área cerebral permanece inactiva, todas colaboran en el procesamiento de la información (Bear et al., 2016); b) el condicionamiento del aprendizaje hasta los tres años es otro error (OECD, 2002), es decir, que las experiencias durante los tres primeros años de vida no determinarán el desarrollo del aprendizaje durante la vida. Se sabe que los mil primeros días de vida corresponden a un período neurobiológico crítico, sustentada esta información en la gran cantidad de nacimiento de neuronas (neurogénesis) y creación de sinapsis (sinaptogénesis) en este período (Alarcón, 2019), sin embargo, se sabe que estos procesos pueden ocurrir durante toda la vida y no son exclusivos de dicho período crítico (Binder \& Scharfman, 2004, Huang \& Reichardt, 2004, Kramer \& Erickson, 2007, Maureira, 2018), además, se deja de lado, desde esta perspectiva, las responsabilidades educativas en adultos y adultos mayores (Bacigalupe, 2020); c) el aprendizaje mejora si se utilizan estrategias acorde al estilo de aprendizaje VAK (visual, auditivo y kinestésico) de los estudiantes (Dunn \& Dunn, 1978). Esta idea se basa en la premisa de que cada persona posee un canal de percepción más desarrollado y a través del cual su aprendizaje sería más eficiente. Sin embargo, no se observan diferencias significativas en el aprendizaje cuando la información se entrega según la vía preferente del estudiante (Riener \& Willingham,
2010). Además, la utilización de la vía de percepción preferente no es un predictor adecuado de un aprendizaje más efectivo (Kirschner, 2017); d) que los estudiantes tengan un hemisferio cerebral predominante es otro neuromito (Ansari, 2008). Esta falacia surge de la creencia de que solo el hemisferio derecho es lingüístico y creativo, y que el hemisferio izquierdo es lógico. Sin embargo, ambos hemisferios están conectados por el cuerpo calloso, lo que provoca un intercambio de información continua, razón por la cual ambos hemisferios no pueden trabajar separados (Kandel et al., 2013).

Entre los estudios sobre la prevalencia de neuromitos en el ámbito educativo, destaca el trabajo de Dekker et al. (2012) donde se mostró una prevalencia de falsas creencias (como los estilos de aprendizaje VAK y la dominancia cerebral) en más del $90 \%$ de profesores encuestados en Inglaterra y Holanda $(n=242)$. Un trabajo de Rato et al. (2013) donde encuestaron a profesores de Portugal $(n=219)$, reveló que el $50 \%$ creía en el estilo de aprendizaje VAK, más del $40 \%$ creía en la dominancia cerebral y el $60 \%$ creía en las inteligencias múltiples. Ferrero et al. (2016) encuestaron a profesores de diferentes regiones de España $(n=284)$ observando que el 94\% creía que ambientes ricos en estímulos ayudaban a desarrollar más el cerebro en preescolares, el 91\% creía en los estilos de aprendizaje VAK y el $67 \%$ en la dominancia cerebral. Bailey et al. (2018) evaluaron a entrenadores/as deportivos de Inglaterra, Irlanda, Escocia y Gales $(n=545)$ mostrando que el 62\% creen en el estilo de aprendizaje VAK, un $42 \%$ cree en la dominancia cerebral y un $22 \%$ cree que usamos solo el 10\% del cerebro.

Un estudio realizado con profesores de primaria, secundaria y educación superior de China $(\mathrm{n}=238)$ reveló que el 97\% cree en los estilos de aprendizajeVAK, el $71 \%$ cree en la dominancia cerebral y el $50 \%$ cree que usamos solo el 10\% del cerebro (Pei et al., 2014). En Latinoamérica, el primer estudio fue realizado por Herculano-Houzel (2002) en una población de Brasil $(n=2158)$ mostrando que los estudiantes de secundaria lograron un $46,3 \%$ de respuestas correctas sobre conocimiento del cerebro, contra un $64,4 \%$ observado en estudiantes universitarios y un $87,1 \%$ en neurocientíficos. Gleichgerrcht et al. (2015) encuestaron a 3451 profesores deArgentina $(n=551)$, Chile $(n=598)$, Perú $(\mathrm{n}=2222)$ y otros países de América Latina $(\mathrm{n}=80)$. Del total, el 66,7\% respondió de manera correcta las preguntas sobre el cerebro, en Perú y Chile menos del $50 \%$ identificó correctamente los neuromitos y en Argentina fue menos del $45 \%$. Un estudio realizado con 
profesores mexicanos de educación infantil y primaria $(n=20)$ reveló que el 29\% creía en que usamos solo el $10 \%$ del cerebro y que escuchar música clásica hace más inteligentes a los niños, y que el $23 \%$ cree en la dominancia cerebral (Medel \& Camacho, 2019). Falquez y Ocampo (2018) encuestaron a estudiantes de pedagogía de Ecuador ( $\mathrm{n}=328)$ reportando que más del 90\% creía en los estilos de aprendizaje VAK y que ambientes ricos en estímulos ayudaban a desarrollar más el cerebro en preescolares. Finalmente, Varas y Ferreira (2017) encuestaron a 91 profesores de establecimientos escolares de Chile mostrando que el 91\% cree en los estilos de aprendizaje VAK y que entornos con mucha estimulación mejora el desarrollo del cerebro de preescolares. Además, un $86 \%$ cree en la dominancia cerebral.

La neurociencia cumple un rol fundamental para las carreras de pedagogía, pues corresponde a conocimientos fundamentales de la comprensión de la naturaleza humana e integralidad desde un enfoque biológico, físico, psíquico e histórico social (Jiménez, et al., 2019). Por ello, la praxis pedagógica necesita hacer suyos los aportes que brinda dicho campo científico para crear espacios educativos de mayor calidad.

Los docentes y estudiantes son agentes de una comunidad académica, y considerando los aportes que la neurociencia puede realizar a la pedagogía, queda claro que el derrumbar los neuromitos entendidos como creencias sin fundamento solido resulta fundamental, ya que permitirá evitar la implementación errónea de esta en los procesos formativos de las distintas disciplinas, como la Educación Física. Es necesario distinguir entre las creencias que aparentan certidumbre acerca del funcionamiento del cerebro y aquello que realmente es información científica útil para la comprensión de los variados fenómenos que acontecen en la educación física.

Considerando los antecedentes expuestos, el objetivo del presente estudio es describir la prevalencia de neuromitos y el conocimiento general de neurociencia de estudiantes de Pedagogía en Educación Física de diversas universidades de Chile. Por otra parte, se evalúan diferentes variables sociodemográficas que podrían explicar la prevalencia de neuromitos y los conocimientos en neurociencia entre los futuros docentes.

\section{Método}

\section{Muestra}

De tipo no aleatorio intencional (Maureira \& Flo- res, 2018), que estuvo constituido por 440 estudiantes de la Carrera de Pedagogía en Educación Física de cinco universidades de Chile. La edad mínima fue de 18 y la máxima de 45 años, con una media de 21.9 3 3.2. Del total de la muestra 143 son mujeres (32.5\%) y 297 son hombres (67.5\%). 114 estudiantes cursaban primer año de la carrera (25.9\%), 84 segundo año (19.2\%), 126 tercer año (28.6\%), 85 cuarto año (19.3\%) y 31 quinto año (7.0\%). Del total, 160 declararon leer habitualmente libros y artículos científicos (36.4\%) y mientras que 280 declararon no hacerlo (63.6\%). Todos los participantes firmaron un consentimiento informado para formar parte del estudio, de manera voluntaria.

\section{Instrumento}

Se aplicó el Cuestionario de prevalencia de neuromitos propuesto por Dekker et al. (2012) en su versión en español (Falquez \& Ocampo, 2018). Este cuestionario está constituido por 32 afirmaciones, 20 de las cuales corresponden a preguntas sobre conocimiento general acerca del funcionamiento del cerebro siendo algunas de ellas verdaderas y otras falsas. Otros 12 ítems corresponden a neuromitos, por lo cual son afirmaciones falsas. Todas las preguntas tienen tres posibles respuestas: verdadero, falso o no lo sé.

\section{Procedimiento}

El cuestionario fue aplicado a los sujetos durante las horas de clases entre las 10:00 y las 13:30 horas. La aplicación tuvo una duración de 15 minutos.

\section{Análisis de datos}

Para el presente estudio se utilizó el programa estadístico IBM SPSS Statistics paraWindows, Versión 25.0. Se aplicó estadística descriptiva con tablas de frecuencia, medias y desviaciones estándar. Se utilizaron tablas cruzadas y pruebas de $\mathrm{X}^{2}$ para comparar los porcentajes de respuestas verdaderas, falsas y no lo sé según sexo, curso y lectura de libros y/o artículos científicos observados en la muestra. Posteriormente se calculó la cantidad de respuestas correctas de los estudiantes y se aplicaron pruebas Kolmogorov-Smirnov (KS) de normalidad de datos entregando un p>.05 razón por la cual se procedió a utilizar estadística inferencial paramétrica, aplicando pruebas t para muestras independientes para comparar por sexo y lectura de textos científicos y ANOVA con pruebas post-hoc de Tukey para comparar por cursos (Maureira, 2017). Se consideró significativo un valor $\mathrm{p}<.05$. 
Tabla 1.

Porcentaje de respuestas a conocimiento general sobre el funcionamiento del cerebro en el total de la muestra.

\begin{tabular}{|c|c|c|c|c|}
\hline tems & Respuesta & Verdadero & Falso & No lo sé \\
\hline Utilizamos nuestro cerebro 24 horas al día & Verdadero & $392(89.1 \%)$ & $24(5.5 \%)$ & $24(5.5 \%)$ \\
\hline 3. El cerebro de los niños es más grande que el de las niñas & Verdadero & $53(12.0 \%)$ & $238(54.1 \%)$ & $149(33.9 \%)$ \\
\hline 5. Cuando se daña un área del cerebro, otra área puede asumir su función & Verdadero & $83(18.9 \%)$ & $269(61.1 \%)$ & $88(20.0 \%)$ \\
\hline El hemisferio izquierdo y derecho del cerebro siempre trabajan juntos & Verdadero & $155(35.2 \%)$ & $195(44.3 \%)$ & $90(20.5 \%)$ \\
\hline 0.El cerebro de niños y niñas se desarrolla al mismo ritmo & Falso & 101.(23.0\%) & $211(48.0 \%)$ & $128(29.1 \%)$ \\
\hline 1. El desarrollo del cerebro termina al mismo tiempo que los estudiantes comienzan la enseñanza media & Falso & $10(2.3 \%)$ & $332(75.5 \%)$ & $98(22.3 \%)$ \\
\hline 3.La información se almacena en una red de células distribuidas en todo el cerebro & Verdadero & $270(61.4 \%)$ & $73(16.6 \%)$ & $97(22.0 \%)$ \\
\hline 4.El aprendizaje se produce por la generación de nuevas células cerebrales & Falso & $150(34.1 \%)$ & $139(31.6 \%)$ & $151(34.3 \%)$ \\
\hline 6.El aprendizaje ocurre por la modificación de las conexiones neuronales del cerebro & Verdadero & $294(66.8 \%)$ & $20(4.5 \%)$ & $126(28.6 \%)$ \\
\hline 7.El buen rendimiento académico puede verse afectado por no tomar desayuno & Verdadero & $319(72.5 \%)$ & $49(11.1 \%)$ & $72(16.4 \%)$ \\
\hline 8.El desarrollo normal del cerebro humano involucra la generación y pérdida de células cerebrales & Verdadero & $280(63.6 \%)$ & $30(6.8 \%)$ & $130(29.5 \%)$ \\
\hline 9. La capacidad mental es hereditaria y no puede modificarse por influencia del ambiente ni de la experiencia & Falso & $21(4.8 \%)$ & $363(82.5 \%)$ & $56(12.7 \%)$ \\
\hline 20.El ejercicio físico vigoroso puede mejorar el desempeño mental & Verdadero & $376(85.5 \%)$ & $15(3.4 \%)$ & $49(11.1 \%)$ \\
\hline $\begin{array}{l}\text { 3.El ritmo circadiano ("reloj biológico") cambia durante la adolescencia, razón por la cual los estudiantes están más cansados durante las primeras horas de } \\
\text { lase de la mañana }\end{array}$ & Verdadero & $203(46.1 \%)$ & $36(8.2 \%)$ & $201(45.7 \%)$ \\
\hline 4.El consumo regular de cafeína reduce la capacidad de atención & Verdadero & $104(23.6 \%)$ & $138(31.4 \%)$ & $198(45.0 \%)$ \\
\hline 6. El reforzamiento constante de ciertos procesos mentales puede cambiar la forma y estructura de ciertas partes del cerebro & Verdadero & $229(52.0 \%)$ & $66(15.0 \%)$ & $145(33.0 \%)$ \\
\hline 7. Cada estudiante muestra preferencias por el modo en que recibe la información (por ejemplo, visual, auditiva, kinestésica) & Verdadero & $418(95.0 \%)$ & $7(1.6 \%)$ & $15(3.4 \%)$ \\
\hline 9. La producción de nuevas conexiones cerebrales puede continuar hasta una edad avanzada & Verdadero & $271(61.6 \%)$ & $54(12.3 \%)$ & $115(26.1 \%)$ \\
\hline 1. Existen períodos sensibles en la infancia durante los cuales es más fácil aprender cosas & Verdadero & $406(92.3 \%)$ & $7(1.6 \%)$ & $27(6.1 \%)$ \\
\hline 2.El cerebro deja de funcionar mientras dormimos & Falso & $6(1.4 \%)$ & $408(92.7 \%)$ & $26(5.9 \%)$ \\
\hline
\end{tabular}

\section{Resultados}

En la tabla 1 se muestran las respuestas sobre el conocimiento general acerca del funcionamiento del cerebro del total de la muestra. Los ítems que lograron un mayor porcentaje de respuestas correctas fueron el ítem 27. Cada estudiante muestra preferencias por el modo en que recibe la información (por ejemplo, visual, auditiva, kinestésica) (95.0\%); ítem 31. Existen períodos sensibles en la infancia durante los cuales es más fácil aprender cosas (92.3\%) e ítem 1. Utilizamos nuestro cerebro 24 horas al día (89.1\%). Los ítems con mayor porcentaje de respuestas incorrectas fueron el ítem 6. Cuando se daña un área del cerebro, otra área puede asumir su función (61.1\%); el ítem 3. El cerebro de los niños es más grande que el de las niñas (54.1\%) e ítem 8. El hemisferio izquierdo y derecho del cerebro siempre trabajan juntos (44.3\%). En relación con las respuestas No lo sé los porcentajes más altos se dieron en el ítem 23. El ritmo circadiano ("reloj biológico») cambia durante la adolescencia, razón por la cual los estudiantes están más cansados durante las primeras horas de clase de la mañana (45.7\%); ítem 24. El consumo regular de cafeína reduce la capacidad de atención (45.0\%) e ítem 14. El aprendizaje se produce por la generación de nuevas células cerebrales (34.3\%).

En la tabla 2 se muestran las respuestas sobre los neuromitos del total de la muestra. Los ítems que lograron un mayor porcentaje de respuestas correctas fue- ron el ítem 4. Si los alumnos no beben cantidades suficientes de agua (6-8 vasos al día), sus cerebros se encogen (56.6\%); ítem 2. Los niños deben adquirir su idioma natal antes de aprender un segundo idioma. Si no lo hacen, ninguno de los dos será completamente adquirido (54.5\%) e ítem 12. Hay períodos críticos en la infancia después de los cuales ciertas cosas ya no se pueden aprender (53.2\%). Los ítems con mayor porcentaje de respuestas incorrectas fueron el ítem 21 . Los entornos que son ricos en estímulos mejoran el cerebro de los niños en edad preescolar (93.4\%); ítem 15. Los individuos aprenden mejor cuando reciben información según su estilo de aprendizaje preferido (por ej. auditivo, visual, kinestésico) (93.2\%) e ítem 30. Breves episodios de ejercicios de coordinación pueden mejorar la integración de la función cerebral hemisférica izquierda y derecha (78.2\%). En relación con las respuestas No lo sé los porcentajes más altos se dieron en el ítem 22. Los niños están menos atentos después de consumir bebidas azucaradas y/o dulces, papas fritas, etc. (35.9\%); ítem 5. Se ha demostrado científicamente que los suplementos de ácidos grasos (omega-3 y omega-6) tienen un efecto positivo en el logro académico (35.5\%) e ítem 4. Si los alumnos no beben cantidades suficientes de agua (6-8 vasos al día), sus cerebros se encogen (34.5\%).

Al comparar el porcentaje de respuestas del conocimiento general sobre el funcionamiento del cerebro según sexo de la muestra se observan diferencias significativas en el ítem 14. El aprendizaje se produce por la

\footnotetext{
Tabla 2.

Porcentaje de respuestas a neuromitos en el total de la muestra.

Items Nordadero Falso No lo sé

2.Los niños deben adquirir su idioma natal antes de aprender un segundo idioma. Si no lo hacen, ninguno de los dos será completamente adquirido $\quad 107(24.3 \%) \quad 240(54.5 \%) \quad 93(21.1 \%)$

4.Si los alumnos no beben cantidades suficientes de agua (6-8 vasos al día), sus cerebros se encogen

5. Se ha demostrado científicamente que los suplementos de ácidos grasos (omega-3 y omega-6) tienen un efecto positivo en el logro académico

7.Solo usamos un $10 \%$ de nuestro cerebro

9.Las diferencias en el dominio hemisférico (cerebro izquierdo, cerebro derecho) pueden ayudar a explicar diferencias individuales entre aprendices

12.Hay períodos críticos en la infancia después de los cuales ciertas cosas ya no se pueden aprender

15. Los individuos aprenden mejor cuando reciben información según su estilo de aprendizaje preferido (por ej. auditivo, visual, kinestésico)

21.Los entornos que son ricos en estímulos mejoran el cerebro de los niños en edad preescolar

22.Los niños están menos atentos después de consumir bebidas azucaradas y/o dulces, papas fritas, etc.

25.Los ejercicios que ponen en práctica la coordinación de las habilidades perceptuales y motrices pueden mejorar las habilidades de lenguaje

28. Los problemas de aprendizaje asociados a las diferencias en el desarrollo del funcionamiento del cerebro no pueden ser mejorados/remediados por la educación

$39(8.9 \%) \quad 249(56.6 \%) \quad 152(34.5 \%)$ $276(62.7 \%) \quad 8(1.8 \%) \quad 156(35.5 \%)$ $183(41.6 \%) \quad 192(43.6 \%) \quad 65(14.8 \%)$ $293(66.6 \%) \quad 27(6.1 \%) \quad 120(27.3 \%)$ $117(26.6 \%) 234(53.2 \%) 89(20.2 \%)$ (12. (11)

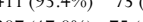
$207(47.0 \%) \quad 75(17.0 \%) \quad 158(35.9 \%)$ $286(65.0 \%) \quad 21(4.8 \%) \quad 133(30.2 \%)$ $310(70.5 \%) \quad 24(5.5 \%) \quad 106(24.1 \%)$ 30. Breves episodios de ejercicios de coordinación pueden mejorar la integración de la función cerebral hemisférica izquierda y derecha $344(78.2 \%) \quad 10(2.3 \%) \quad 86(19.5 \%)$
} 
generación de nuevas células cerebrales, donde los hombres presentan mayor cantidad de respuestas correctas (mujeres $=27.3 \%$; hombres $=33.7 \%$; $\mathrm{p}=.003) ; \mathrm{y}$ en el ítem 17. El buen rendimiento académico puede verse afectado por no tomar desayuno, donde los hombres presentan mayor cantidad de respuestas correctas (mujeres $=67.1 \%$; hombres $=75.1 \%$; $\mathrm{p}=.030)$.

Tabla 3.

Diferencias en el porcentaje de respuestas correctas a conocimiento general sobre el funcionamiento del cerebro en el total de la muestra, según lectura de libros científicos y/o artículos científicos.

\begin{tabular}{lccc}
\hline Items & Leen & No leen & Valor $p$ \\
\hline 11.El desarrollo del cerebro termina al mismo tiempo que los estudiantes comienzan la enseñanza media & $77.5 \%$ & $74.4 \%$ & .031 \\
14.El aprendizaje se produce por la generación de nuevas células cerebrales & $35.6 \%$ & $29.3 \%$ & .045 \\
16.El aprendizaje ocurre por la modificación de las conexiones neuronales del cerebro & $37.5 \%$ & $32.1 \%$ & .022 \\
17.El buen rendimiento académico puede verse afectado por no tomar desayuno & $76.9 \%$ & $70.0 \%$ & .046 \\
18.El desarrollo normal del cerebro humano involucra la generación y pérdida de células cerebrales & $68.8 \%$ & $60.7 \%$ & .036 \\
19.La capacidad mental es hereditaria y no puede modificarse por influencia del ambiente ni de la experiencia & $83.8 \%$ & $81.8 \%$ & .029 \\
20.El ejercicio físico vigoroso puede mejorar el desempeño mental & $92.5 \%$ & $81.4 \%$ & .006 \\
29.La producción de nuevas conexiones cerebrales puede continuar hasta una edad avanzada & $70.0 \%$ & $56.8 \%$ & .015 \\
\hline
\end{tabular}

Al comparar el porcentaje de respuestas a neuromitos según el sexo de la muestra se aprecian diferencias significativas solo en el ítem 2. Los niños deben adquirir su idioma natal antes de aprender un segundo idioma. Si no lo hacen, ninguno de los dos será completamente adquirido, donde las mujeres presentan mayor cantidad de respuestas correctas (mujeres $=65.7 \%$; hombres $=49.2 \% ; \mathrm{p}=.002$ ).

Al comparar el porcentaje de respuestas del conocimiento general sobre el funcionamiento del cerebro según curso de la muestra se observan diferencias significativas solo en el ítem 2. Cuando se daña un área del cerebro, otra área puede asumir su función, donde primer y quinto año presentan mayor cantidad de respuestas correctas en comparación con cuarto año $(\mathrm{p}=.049)$; en el ítem 3. El cerebro de los niños es más grande que el de las niñas, donde quinto año presenta más respuestas correctas en comparación con primer, segundo y cuarto año $(\mathrm{p}=.034)$; en el ítem 14. El aprendizaje se produce por la generación de nuevas células cerebrales, donde los estudiantes de quinto año presentan menor cantidad de respuestas correctas que el resto de los cursos $(\mathrm{p}=.013)$; y en el ítem 29. La producción de nuevas conexiones cerebrales puede continuar hasta una edad avanzada, donde los estudiantes de tercer año poseen mayor cantidad de respuestas correctas en comparación con quinto año $(\mathrm{p}=.018)$.

Al comparar el porcentaje de respuestas a neuromitos según el curso solo se observan diferencias en el ítem 12. Hay períodos críticos en la infancia después de los cuales ciertas cosas ya no se pueden aprender, donde quinto año presenta la menor cantidad de respuestas correctas $(p=.020)$.

En la tabla 3 se aprecian las diferencias en el porcentaje de respuestas correctas del conocimiento general sobre el funcionamiento del cerebro entre los estudiantes que declaran habitualmente leer libros científicos y/o artículos científicos y los que declaran no hacerlo. Es posible notar que en los ocho ítems que presentan diferencias, siempre el mayor porcentaje de respuestas correctas es obtenido por los estudiantes que declaran leer este tipo de material.
Al comparar el porcentaje de respuestas a neuromitos entre los estudiantes que declaran habitualmente leer libros científicos y/o artículos científicos se aprecian diferencias significativas en el ítem 2. Los niños deben adquirir su idioma natal antes de aprender un segundo idioma. Si no lo hacen, ninguno de los dos será completamente adquirido, donde los estudiantes que afirman leer material científico presentan mayor cantidad de respuestas correctas $\left(\mathrm{No}_{\mathrm{O}}=49,6 \% ; \mathrm{Si}=63,1 \% ; \mathrm{p}=.007\right)$; en el ítem 4. Si los alumnos no beben cantidades suficientes de agua (6-8 vasos al día), sus cerebros se encogen, donde los estudiantes que afirman no leer material científico presentan mayor cantidad de respuestas correctas $(\mathrm{No}=57.9 \%$; $\mathrm{Si}=54.4 \%$; $\mathrm{p}=.023)$; y en el ítem 22. Los niños están menos atentos después de consumir bebidas azucaradas y / o dulces, papas fritas, etc., donde los estudiantes que afirman no leer material científico presentan mayor cantidad de respuestas correctas $(\mathrm{No}=18.9 \% ; \mathrm{Si}=13.8 \% ; \mathrm{p}=.039)$.

En la tabla 4 se muestra la cantidad de respuestas correctas del conocimiento general sobre el funcionamiento del cerebro y de los neuromitos según sexo, curso y lectura de libros y/o artículos científicos. La media general de respuestas correctas en el conocimiento general del cerebro fue de $12.1 \pm 2.8$ de un máximo posible de 20 y en los neuromitos fue de $2.5 \pm 1.4$ de un máximo posible de 12. En el caso del conocimiento general del cerebro, el 1.2\% de la muestra respondió correctamente 5 preguntas o menos, el 25.6\% respondió correctamente entre 6 y 10 preguntas, el 63.8\% respondió correctamente entre 11 y 15 preguntas y el $9.3 \%$ respondió correctamente 16 o más preguntas. Por lo tanto, el 73,1\% de la muestra contesto correctamente al menos el 50\% del cuestionario sobre conocimiento general del cerebro.

En el caso de los neuromitos el 5.9\% de la muestra no presenta ninguna respuesta correcta, el $72.2 \%$ respondió correctamente entre 1 y 3 preguntas, el $21.6 \%$ respondió correctamente entre 4 y 6 preguntas y el $.2 \%$ respondió correctamente 7 o más preguntas. Por 
lo tanto, solo el $2.7 \%$ de la muestra contesto correctamente al menos el 50\% del cuestionario de neuromitos, lo que da cuenta de una alta prevalencia de estas creencias.

El conocimiento general del cerebro muestra diferencias a favor de los estudiantes que declaran leer libros y/o artículos científicos ( $\mathrm{p}=.000)$, en tanto, no se observan diferencias significativas de neuromitos entre mujeres y hombres, por curso y entre los que leen textos científicos y los que no.

\begin{tabular}{|c|c|c|}
\hline & Conocimiento general del cerebro & Neuromitos \\
\hline Total & $12.1 \pm 2.8$ & $2.5 \pm 1.4$ \\
\hline Mujeres & $11.8 \pm 2.6$ & $2.5 \pm 1.4$ \\
\hline Hombres & $12.2 \pm 2.9$ & $2.5 \pm 1.4$ \\
\hline Valor $p$ & .273 & .583 \\
\hline $1^{\circ}$ año & $11.4 \pm 2.9$ & $2.4 \pm 1.5$ \\
\hline $2^{\circ}$ año & $12.1 \pm 2.8$ & $2.6 \pm 1.4$ \\
\hline $3^{\circ}$ año & $12.4 \pm 2.9$ & $2.5 \pm 1.3$ \\
\hline $4^{\circ}$ año & $12.4 \pm 2.6$ & $2.8 \pm 1.4$ \\
\hline $5^{\circ}$ año & $11.9 \pm 2.6$ & $2.0 \pm 1.4$ \\
\hline Valor $p$ & $.038 *$ & .080 \\
\hline Tukey & $3^{\circ}>1^{\circ}$ & - \\
\hline Si lee & $12.8 \pm 2.7$ & $2.6 \pm 1.2$ \\
\hline No lee & $11.7 \pm 2.8$ & $2.5 \pm 1.5$ \\
\hline Valor $p$ & $.000 * *$ & .428 \\
\hline
\end{tabular}

\section{Discusión}

El objetivo del presente estudio fue describir la prevalencia de neuromitos y el conocimiento general de neurociencia de estudiantes de Pedagogía en Educación Física de diversas universidades de Chile. El tema es de gran interés ya que, como lo mencionan Torrijos-Muelas et al., (2021), han transcurrido casi dos décadas desde los primeros reportes de neuromitos en el área de la educación; no obstante, éstos siguen concitando gran atención, reportándose un incremento de las publicaciones que demuestran lo diseminados que están los neuromitos en todos los niveles educativos a nivel mundial (Torrijos-Muelas et al., 2021; van Dijk \& Lane, 2020).

Para la presente investigación, en 12 de los 20 ítems del cuestionario de conocimiento general del cerebro, la muestra obtuvo más de un 60\% de respuestas correctas, resaltando conocimientos sobre las preferencias en el modo de recibir la información y la existencia de períodos sensibles donde es más fácil aprender, en el cual las respuestas correctas superaron el 90\%. Lo anterior puede ser contrastado con lo reportado por Chiang et al., (2020) quienes, luego de estudiar a 968 profesores en formación en Hong Kong, reportan solo un 29,2 $\%$ de respuestas correctas respecto a la existencia de períodos sensibles donde se hace más fácil aprender. Sin embargo, encontraron un porcentaje similar (94,3 \%) a lo reportado en el presente estudio en relación con la existencia de preferencias que tienen los individuos respecto a la manera de recibir la información. Un porcentaje levemente inferior (84,2 \%), pero en la misma tendencia, también ha sido reportado recientemente por Carter et al. (2020) luego de haber encuestado a 1359 educadores en formación australianos. En relación con los neuromitos, los más difundidos en esta muestra son los estilos de aprendizaje VAK y los efectos positivos de los entornos enriquecido en la edad preescolar, ambos con más del 90\% de prevalencia. Estos resultados son similares a los encontrados por Dekker et al. (2012) en profesores de Inglaterra y Holanda, Ferrero et al. (2016) en profesores españoles, Pei et al. (2014) en profesores de China, Falquez y Ocampo (2018) en estudiantes de pedagogía de Ecuador, Hughes et al. (2020) entre educadores australianos y por los autores Varas y Ferreira (2017) en profesores de Chile.

En términos generales, es posible asumir que los conocimientos sobre el cerebro son similares en mujeres y hombres de esta muestra. Al comparar por cursos se presentan diferencias solo en cinco de los 32 ítems del cuestionario, pero sin ningún patrón aparente de prevalencia en algún curso de la formación pedagógica de profesor de Educación Física.

Los resultados obtenidos en el presente estudio entregan antecedentes de discusión a una temática muy activa actualmente, que guarda relación con la necesidad de incluir en las innovaciones curriculares de los planes de formación de los futuros docentes aquellos contenidos relacionados con la neurociencias, así como el prevenir y desarraigar algunos neuromitos fuertemente internalizados entre educadores en formación, profesores en ejercicio e, incluso, directivos educacionales (Hughes et al., 2020; van Dijk \& Lane, 2020). En este sentido, Maureira y Flores (2020) analizaron las mallas académicas de las carreras de pedagogía en Educación Física de Chile, observando la falta de asignaturas relacionadas con neurociencias y educación. Esto último podría explicar, en parte, la alta prevalencia de neuromitos en estos estudiantes.

Por otra parte, se ha reportado recientemente que los profesores en formación austríacos no tienen un mejor o peor desempeño académico si creen o no en neuromitos (Krammer et al., 2020), pero estos mismos investigadores argumentan que se debe trabajar a favor de disminuir la creencia en neuromitos pues no es compatible con una formación y práctica docente basada en la evidencia (Krammer et al., 2020). Algunas investigaciones han reportado que los neuromitos permean bastante en los contextos educativos y son muy 
persistentes y podrían estar más difundidos de lo que se piensa, incluso a nivel de directivos, líderes educacionales, y académicos universitarios (van Dijk \& Lane, 2020). Frente a esta realidad, se debe incluir eficazmente a la neurociencia como parte de la formación inicial del profesorado, pero para ello es necesario tener una comprensión más amplia de la alfabetización en neurociencia entre los futuros profesores y de sus percepciones sobre la neurociencia en la educación. Esta comprensión es útil para el desarrollo y la planificación de futuros programas de formación docente (Ching et al., 2020). Para lograr esto, Hughes et al., (2020) recomiendan propender a una asociación más sólida con los neurocientíficos y así abordar el complejo problema de separar los hechos cerebrales de las ficciones y brindar finalmente un mejor apoyo formativo a los maestros. Respecto a lo anterior, es importante rescatar la idea de que los avances en las ciencias permiten (y han permitido históricamente) derribar verdades asumidas (mitos) en distintas épocas y no perpetuarlos (Bacigalupe, 2020). Para esto, es relevante plantear una formación adecuada y seria en neurociencias entre los futuros docentes, en particular, en los de educación física, ya que la ventaja de distinguir los neuromitos de la neurociencia en la práctica pedagógica de esta disciplina, es que se evita caer en reduccionismos y en aplicaciones excesivamente entusiastas, poco exactas y que eventualmente podrían ser perjudiciales.

\section{Conclusión}

En el presente estudio los neuromitos más prevalentes estuvieron relacionados con los entornos ricos en estímulos, los suplementos de ácidos grasos (omega-3 y omega-6), los ejercicios de coordinación y la integración hemisférica y los estilos de aprendizaje VAK. No se encontraron diferencias significativas entre los conocimientos acerca del cerebro, neuromitos y las variables sociodemográficas estudiadas en la muestra. Los resultados observados en la presente investigación son similares a los reportados en otros estudios, en estudiantes y profesores, tanto en Chile como a nivel internacional.

Sería recomendable estudiar la prevalencia de neuromitos en otras carreras de pedagogías. También sería interesante relacionar los conocimientos generales del cerebro en los profesores universitarios que imparten las clases en pedagogía, para determinar la posible influencia de estos en la adquisición de neuromitos en los futuros docentes. Por otra parte, podría realizar- se esta investigación experimental, donde los estudiantes pudiesen acceder a un curso o taller de neurociencia aplicada a la educación y también crear un instrumento con ítems disciplinares para poder erradicar neuromitos propios de la educación física.

\section{Agradecimientos}

Al Centro de Investigación en Educación-UBO y al fondo «XIV Concurso de Investigación en Docencia Universitaria UBO 2020» proyecto $\mathrm{N}^{\circ} \mathrm{UBO} / \mathrm{VVCMEI}$ 20203

\section{Referencias}

Alarcón, O. (2019). Neurodesarrollo en los primeros 1.000 días de vida. Rol de los pediatras. Revista Chilena de Pediatría, 90(1), 11-16. https://dx.doi.org/10.32641/ rchped.v90i1.1035

Ansari, D. (2008). The brain goes to school: Strehgthening the education-neuroscience connection. Education Canada, 48(4), 6-10.

Aparicio, X. (2009). Neurociencias y la transdisciplinariedad en la educación. Revista Universitaria de Investigación y Diálogo Académico, 5(2), 1-21.

Arias, D. (2017). Análisis de neuroeconomía como nuevo paradigma en la ciencia económica. Ciencias Económicas, 2, 107-119.

Bacigalupe, M. (2020) Los estudios del comportamiento humano en la construcción de la neurociencia educacional. Interdisciplina, 8(22): 223-245. https://doi.org/ 10.22201/ceiich.24485705e.2020.22.76427

Bailey, R., Madigan, D., Cope, E. \& Nicholls, A. (2018). The prevalence of pseudoscientific ideas and neuromyths among sports coaches. Frontiers in Psychology, 9, 641. https: / /doi.org/10.3389/fpsyg.2018.00641

Bear, M., Connors, B. \& Paradiso, M. (2016). Neurociencia, la exploración del cerebro. Wolters Kluver

Binder, D. \& Scharfman, H. (2004). Brain-derived neurotrophic factor. Growth Factors, 22, 123-131. https:/ /doi.org/10.1080/08977190410001723308

Campos,A. (2010). Neurociencia: uniendo las neurociencias y la educación en la búsqueda del desarrollo humano. $L a$ Educación, 143, 1-14.

Carter, M., Van Bergen, P., Stephenson, J., Newall, C. \& Sweller, N. (2020). Prevalence, Predictors and Sources of Information Regarding Neuromyths in an Australian Cohort of PreserviceTeachers. Australian Journal of Teacher Education, 45(10). 95-113. http:/ /dx.doi.org/10.14221/ ajte. $2020 \mathrm{v} 45 \mathrm{n} 10.6$ 
Ching, F., So, W., Lo, S. K., \& Wong, S. (2020). Preservice teachers' neuroscience literacy and perceptions of neuroscience in education: Implications for teacher education. Trends in Neuroscience and Education, 21, 100144. https: / /doi.org/10.1016/j.tine.2020.100144

Dekker, S., Lee, N., Howard-Jones, P. \& Jolles, J. (2012). Neuromyths in education: Prevalence and predictors of misconceptions among teachers. Frontiers in Psychology, 3,429.https: / / doi.org/10.3389/fpsyg. 2012.00429

Dunn, R. \& Dunn, K. (1978). Teaching students through their individual learning styles: A practical approach. Prentice Hall.

Falquez, J. \& Ocampo, J. (2018). Del conocimiento científiCo al malentendido. Prevalencia de neuromitos en estudiantes ecuatorianos. Revista Iberoamericana de Educación, 78(1), 87-106.

Ferrero, M., Garaizar, P. \& Vadillo, M. (2016). Neuromyths in education: prevalence among spanish teachers and an exploration of cross cultural variation. Frontiers in Human Neuroscience, 10, 496. https://doi.org/10.3389/ fnhum.2016.00496

Geake, J. (2008). Neuromythologies in education. Educational Research, 50(2), 123-133. https://doi.org/10.1080/ 00131880802082518

Gleichgerrcht, E., Luttges, B. L., Salavarezza, F. \& Campos, A. L. (2015). Educational neuromyths among teachers in latin america. Mind, Brain, and Education, 9(3), 170-178. https: / /doi.org/10.1111/mbe.12086

Herculano-Houzel, S. (2002). Do you know your brain? a survey on public neuroscience literacy at the closing of the decade of the brain. Neuroscientist, 8(2), 98-110.https:/ /doi.org/10.1177/107385840200800206

Huang, E. \& Reichardt, L. (2004). Neurotrophins: roles in neuronal development and function. Annual Review of Neuroscience, 24, 677-736. https://doi.org/10.1146/ annurev.neuro.24.1.677

Hughes, B., Sullivan, K. A., \& Gilmore, L. (2020). Why do teachers believe educational neuromyths? Trends in Neuroscience and Education, 21, 100145. https: / / doi.org/ 10.1016/j.tine.2020.100145

Jiménez, E., López, M. \& Herrera, D. (2019). La neurociencia en la formación inicial de docentes. Revista Conrado, 15(67), 241-249. https:/ /doi.org/10.22206/ cyed.2018.v2i3.pp11-25

Kandel, E., Schwartz, J., Jessel, T., Siegelbaum, S. \& HudsPeth,A. (2013). Principles of neural science. McGrawHill.

Kirschner, P. (2017). Stop propagating the learning styles myth. Computers \& Education, 106, 166-171. https: / / doi.org/10.1016/j.compedu.2016.12.006

Kramer, A \& Erickson, K. (2007). Capitalizing on cortical plasticity: influence of physical activity on cognition and brain function. Trends Cognitive Science, 11(8), 342-348. https: / /doi.org/10.1016/j.tics.2007.06.009

Krammer, G., Vogel, S. and Grabner, R. (2021), Believing in neuromyths makes neither a bad nor good student teacher: the relationship between neuromyths and academic achievement in teacher education. Mind, Brain and Education, 15, 54-60. https://doi.org/10.1111/ mbe. 12266

Markram, H. (2006). The blue brain project. Nature Reviews Neuroscience, 7(2), 153-160. https://doi.org/10.1038/ nrn1848

Maureira, F. (2017). Estadística para educación fisica. Bubok Publishing.

Maureira, F. (2018). Principios de neuroeducación fisica. Bubok Publishing.

Maureira, F. \& Flores, E. (2018). Manual de investigación cuantitativa. Bubok Publishing.

Medel, M. \& Camacho, J. (2019). La neurociencia aplicada en el ámbito educativo. El estudio de los neuromitos. International Journal of New Education, 3, 69-83. https: / / doi.org/10.24310/IJNE2.1.2019.6559

OECD (2002). Understanding the brain:Towards a new learning science. OECD.

Pallarés-Domínguez, D. (2016). Neuroeducación en diálogo: neuromitos en el proceso de enseñanza-aprendizaje y en la educación moral. Pensamiento, 72(273), 941-958.

Rato, J., Abreu, A. \& Castro, A. (2013). Neuromyths in education: What is fact and what is fiction for Portuguese teachers? Educational Research, 55(4), 441-453. https: / / doi.org/10.1080/00131881.2013.844947

Riener, C. \& Willingham, D. (2010). The myth of learning styles. Change: The Magazine of Higher Learning, 42(5), 3235.

Torrijos-Muelas, M., González-Víllora, S., \& BodoqueOsma, A. R. (2021). The persistence of neuromyths in the educational settings: a systematic review. Frontiers in Psychology, 11, 591923. https://doi.org/10.3389/ fpsyg.2020.591923

van Dijk, W. \& Lane H. B. (2020). The brain and the US education system: Perpetuation of neuromyths. Exceptionality, A Special Education Journal,28, 16-29. https: / /doi.org/10.1080/09362835.2018.1480954

Varas, P. \& Ferreira, R. (2017). Neuromitos de los profesores chilenos: orígenes y predictores. Estudios Pedagógicos, 43(3), 341-360. 